\title{
Transmission Capacity Of D2D Communication Under Cellular Networks
}

\author{
Xianghai Xü ${ }^{1}$, Jun Sun ${ }^{1}$,Shixiang Shao ${ }^{1}$ \\ ${ }^{1}$ Jiangsu provincial Key Laboratory of Wireless Communication, Nanjing University of Posts and Telecommunications, \\ JiangSu province, China \\ axuxianghai@126.com \{sunjun, shaosx\}@njupt.edu.cn
}

\begin{abstract}
Device-to-Device communication is introduced in cellular system to make full use of the spectrum resources. In order to study the D2D transmission capacity when the D2D multiplexing orthogonal and non-orthogonal of the resource sharing mode, the paper studies D2D system interference in LTE cellular networks. Due from cellular edge user vulnerability, D2D reuse non-orthogonal resources will bring a larger outage probability. In this paper, The D2D reuse resources of cellular users near the base station, which bring greater improvements in the outage probability of cellular users, and then calculated the outage probability and the transmission capacity of D2D in cell system. Found through simulation comparison, It is proximate between theoretical calculations and simulation values when multiplexed the preferred regional cellular user resources.

Index Terms - D2D LTE Transmission Capacity.
\end{abstract}

\section{Introduction}

With the development of mobile communication, LTE is gradually entering the commercial [1], in order to take full advantage of the cellular spectrum resources, a new communications technology D2D (Device-to-Device) [2] [3] communication attracted widespread attention. D2D communication technology is a means of communication where two users establish a direct link without the need to go through the forwarding by the base station. It possible to increase the spectral efficiency of a cellular communication system, to reduce the terminal transmission power, to some extent solve the problem of the lack of a wireless communication system spectrum resources, Compared to the same technology such as unlicensed spectrum application, D2D communication can reduce the base station burden of data forwarding, so as to enhance the throughput of the entire system. Adjustments by multiplexing cell resources to achieve efficient spectrum utilization, thereby improving communication speed and communication quality of the whole cell, the interference generated by the D2D can be controlled by resources allocation of the base station [4], and transmitter power [5] control. Of course, D2D communication, there are some problems [6], Such as how to establish a mechanism to establish end-to-end communication, how to effectively coordinate the cellular network with D2D communications between a pair of interference, as well as relay transmission, namely, how to switch between D2D and cellular network. Klaus Doppler [7] proposed three models to the D2D resources allocation in LTEAdvanced network: a dedicated resource model, the cellular mode and multiplexed mode,. Multiplexed mode contains of cellular users Uplink or Downlink resource reuse. Multiplexing cellular uplink resource would cause interference to base station, the multiplexed downlink resource would cause interference to cellular user. Some scholars have suggested that joined cooperative relay through research to increase the range of D2D communication applications.[8] proposed to select the appropriate user in the cell as a communications relay, to help users achieve communication between the base station. Three relay node approach are $\mathrm{AF}$ (Amplify and Forward)、 DF (Detect and Forward) 、 CC(Coded Cooperation) 。 Transmission capacity is the maximum node density that system can withstand beyond certain outage probability. Since S.Weber proposed the concept of transmission capacity [9], In the past few years, many associated with the study. J.Lee proposed a method to calculate the transmission capacity of the two networks in cognitive radio networks[10], when the distance between the pair users of primer network or secondary network is fixed, derived the optimal power ratio of the two networks and the distribution density of the secondary network, thus more succinct expression of the secondary network transmission capacity is got, On this basis, Ziyang Liu [11] introduce D2D as a secondary network to the primary network where D2D simultaneously share the spectrum resources of the two the primary networks, but the whole power allocated to two bands remain unchanged, and draw optimal power ratio, and the optimal D2D node density, and thereby infer D2D transmission capacity. The same time, as the basis of [9],[12] solve the underlay and overlay of the transmission capacity, and compared t system capacity with capacity domain, this is more similar with [13]. Rahul Vaze study the transmission capacity of the multi-antenna[14], the primary network is only a single antenna, while the secondary network is a multi-antenna, by draw the optimal STDOF and SRDOF and discussed to part of SRDOF to remove interference so as to maximize the transmission capacity of the secondary AdHoc network . Rahul Vaze [15] studied the multi-antenna interference cancellation and traffic adaptation literature techniques, so as to enhance the

\footnotetext{
*This work is partially supported by 973 Program[No.2013CB329005];National Important Special Project[No.2012ZX03003011-005]; National Important Special Project[2012ZX03001008-003];JiangSu Province Special Project[No.BK2011027];School Project[No.NY210007].
} 
transmission capacity. Upper bounds on the D2D.Transmission capacity of multi-hop is studied[16], where potential sourcedestination paths are dynamically selected from a pool of randomly located relays, and a closed-form lower bound on the outage probability is derived in terms of the expected number of potential paths.

\section{System Model And Interference Analyse}

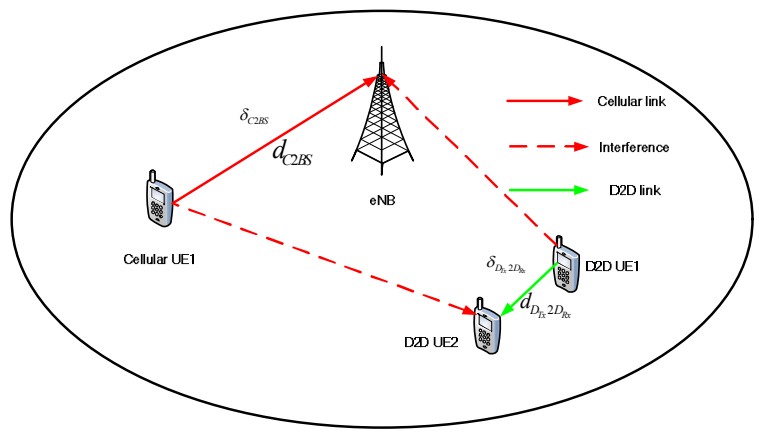

Fig

ure.1. An example of coexisting of two different systems (the nodes of cellular UE and D2D UE).

Figure 1 shows a cellular system which conclude D2D distribution, eNB in the cell center is the base station, which is FDD in the work.D2D users can communication directly under the coordination of eNB, and sharing resources with cellular users, we focuses D2D multiplexing the uplink resource of the cellular system, due to the orthogonality of the uplink resource system, for simplicity, nodes are fixed.

The user kind in cellular system is indicated for $\Phi=\{C D\}$, $\mathrm{C}$ is cellular communication and D is D2D communication.It is also assumed that the distribution of D2D nodes of system follows a stationary Poisson point process (PPP) with density of $\lambda_{D}$ in the finite two-dimensional plane. The channel model includes path loss and Rayleigh fading. Therefore, the power of the node $\mathrm{i}$ received from $\mathrm{j}$ can be expressed as: $P_{i} \delta_{i j}\left|X_{\mathrm{ij}}\right|^{-\alpha}, \mathrm{i}$ 、 $\mathrm{j} \in \Phi, P_{i}$ is the power of node $\mathrm{i}, \delta_{i j}$ is the Rayleigh fading index between $\mathrm{i}$ and $\mathrm{j}$, and it has an exponential distribution with unit mean, $\left|X_{i j}\right|$ is the distance between node $\mathrm{i}$ and $\mathrm{j}, \alpha$ is the pathloss exponent.

Interference in the system is mainly divided into two categories: 1、D2D user multiplex orthogonal resources to the cellular user, means that the D2D communication will not cause interference to cellular users, interference exists only between the D2D pairs using the same resource.2、D2D user multiplex the same resources with cellular user,so the eNB receive interference from D2D transmitter, at the same time D2D receiver receives interference from cellular user. The node of interference can be expressed as: $\Pi_{j}=\left\{\left(X_{j i}, \delta_{j i}\right)\right\}, \forall j \in \Phi$.so the signal to interference-plus-noise ratio (SINR) at the typical receiver $\mathrm{k}$ becomes[10]

$$
\operatorname{SINR}_{k}=\frac{P_{k} \delta_{k 0} R_{k}^{-\alpha}}{\sum_{j \in \Phi} \sum_{X j i \in \Pi j} P_{j} \delta_{j i}\left|X_{j i}\right|^{-\alpha}+N_{0}}
$$

where $\delta_{j i}$ is the fading factor on the power transmitted from the desired transmitter to the receiver, $N_{0}$ is the thermal noisepower, spectrum sharing systems are interference-limited systems, so the thermal noise is negligible in regimes of interest. So the SIR can be used instead of SINR, which allows simplification to :

$$
S I R_{k}=\frac{\delta_{k 0} R_{k}^{-\alpha}}{I_{k}}
$$

$I_{k}=\sum_{j \in \Phi} I_{k j}$ Where $I_{k j}$ is the sum of the power normalized interference from transmitting nodes of system $\mathrm{j}$ to the receiver of system $\mathrm{k}$, which is defined as $I_{k j}=\left(P_{i} / P_{k}\right) \sum_{X_{J I} \in \Pi_{j}} \delta_{j i}\left|X_{j i}\right|^{-\alpha}$,for QOS of system k(D2D or cellular users), SIR required to meet a certain threshold: $S I R_{k} \geq V_{k}$, where $V_{K}$ is target SIR, then the probability of successful transmission can be defined as:

$$
\begin{aligned}
P\left(S I R_{k}>V_{k}\right) & =P\left(\delta_{k 0} \geq V_{k} R_{k}^{\alpha} I_{k}\right) \\
& =\int_{0}^{\infty} P\left\{\delta_{k 0} \geq V_{k} R_{k}^{\alpha} I_{k}\right\} f_{I_{k}}(I) d I \\
& =\psi_{I_{k}}\left(V_{k} R_{k}^{\alpha}\right)
\end{aligned}
$$

$\delta_{k 0}$ is exponentially distributed, then $\psi_{I_{K}}\left(V_{k} R_{k}^{\alpha}\right)$ is the Laplace transform[17] of $f_{I_{k}}(I)$, which can be expressed for $E\left[e^{-I_{k} \eta}\right], I_{k}=\sum_{j \in \phi} I_{k j}$, the interference $I_{k j}$ is independent to each other, $E\left[e^{-I_{k} \eta}\right]=\prod_{j \in \phi} E\left[e^{-I_{k j} \eta}\right]$, then the probability of successful transmission can be changed to

$$
\psi_{I_{\mathrm{k}}}\left(V_{k} R_{k}^{\alpha}\right)=\prod_{j \in \phi} \psi_{I_{K_{j}}}\left(V_{k} R_{k}^{\alpha}\right)
$$

Especially, When the interfering nodes is Poisson point distributed:

$$
\begin{aligned}
\psi_{I j}\left(\mathrm{v}_{k} \mathrm{R}_{k}^{\alpha}\right) & =\exp \left\{-\lambda_{j} \int_{R^{2}} 1-E\left[e^{-V_{k} R_{k}^{\alpha} \delta \mid X \digamma^{\alpha}}\right] d x\right\} \\
& =\exp \left\{-\lambda_{j} \int_{R^{2}} \frac{V_{k} R_{k}^{\alpha} \delta|X|^{-\alpha}}{1+V_{k} R_{k}^{\alpha} \delta|X|^{-\alpha}} d x\right\} \\
& =\exp \left\{-C_{\alpha} R_{k}^{2} V_{K}^{2 / \alpha} \gamma_{k j} \lambda_{j}\right\}
\end{aligned}
$$


$\gamma_{k j}=\left(P_{j} / P_{k}\right)^{2 / \alpha}$ is power ratio, where $P_{j} 、 P_{\mathrm{k}}$ are power of system $\mathrm{j}$ and $\mathrm{k}, C_{\alpha}=(2 \pi / \alpha) \Gamma(2 / \alpha) \Gamma(1-2 / \alpha)$, where $\Gamma(x)=\int_{0}^{\infty} y^{x-1} e^{-y} d y$ is gamma function. Therefore, the success transmission probability of system $\mathrm{k}$ is:

$$
P\left(S I R_{K} \geq V_{K}\right)=\exp \left\{-\kappa_{\kappa} \sum_{j \in \Phi} \gamma_{k j} \lambda_{j}\right\}
$$

where $\kappa_{\kappa}=C_{\alpha} R_{\mathrm{k}}^{2} V_{k}^{2 / \alpha}$

\section{D2D Multiplex Orthogonal Resource}

D2D user multiplex orthogonal resources to the cellular user, means that $\mathrm{D} 2 \mathrm{D}$ use the resources which is not occupied by cellular users, so interference exist only among D2D users, then the outage probability of reference receiver is

$$
q(\lambda)=1-\exp \left\{-\frac{2 \pi^{2}}{\alpha \sin (2 \pi / \alpha)} R_{k}^{2} V_{k}^{2 / \alpha} \lambda\right\}
$$

at the same time Transmission capacity can be expressed as

$$
C(\epsilon)=\lambda(1-\in)
$$

Where $\in$ is the outage probability and $\lambda$ is the density of the D2D nodes, we set that $\alpha=4, R_{k}=10, V_{k}=10$, Figure 2 shows the relation between D2D density and the outage probability, Figure 3 is the relation between D2D density and transmission capacity, we can find in Figure that the maximum of transmission capacity when the density is about $10^{-3}$, and the outage probability is 0.5 , which is Unacceptable for systems, so the upper bound of outage probability should be limited in the system, for example:0.1.

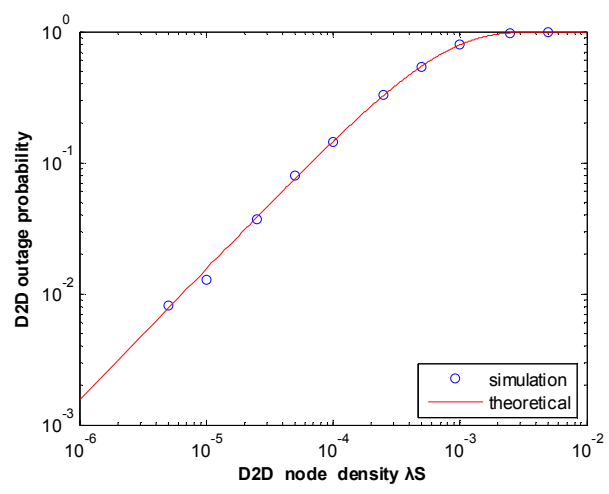

Fig.2. The D2D outage probability accroding to D2D density, where $a=4$, $\mathrm{R}_{\mathrm{k}}=10, \mathrm{~V}_{\mathrm{k}}=10$.

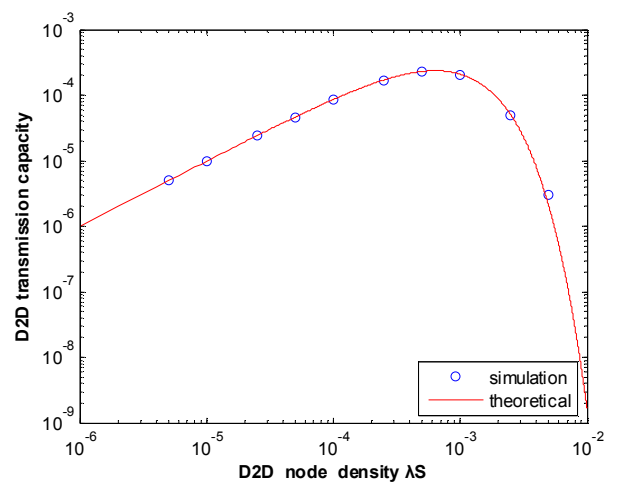

Fig.3. The D2D transmission capacity accroding to D2D density.

\section{D2D Multiplex Non-orthogonal Resource}

When D2D multiplexing non-orthogonal resource of cellular system, the D2D inevitably interfere with cellular systems, it is more complex than multiplexing orthogonal resource, for D2D users, the interference is from D2D and cellular users which multiplex the same resource; for cellular users, the interference is only from D2D which multiplex the same resource. Now we analyze the impact of the cellular user distance from the base station for the outage probability of cellular users, we set that $\mathrm{Pc} / \mathrm{Pd}=100, \mathrm{Pc}$ is power of cellular transmitter, and $\mathrm{Pd}$ is power of D2D transmitter, target SIR is $10 \mathrm{db}$, the distance of D2D is $10 \mathrm{~m}, \mathrm{~d}_{\mathrm{cu}}$ is the distance of the base station to cellular user. We can know that the outage probability of the cellular user is:

$$
\begin{aligned}
& P\left(\operatorname{SIR}_{c u} \leq v_{c u} \mid D_{c u}=d_{c u}\right) \\
= & 1-\exp \left\{-\frac{\pi^{2} d_{c u}^{2} v_{c u}^{2 / \alpha}}{2} \gamma_{C D} \lambda_{D}\right\}
\end{aligned}
$$

Figure 4 shows different outage probability of cellular user with $\mathrm{d}_{\mathrm{cu}}=20,50,100$, Obviously, with the distance from the base station farther, the outage probability become much more higher, as a result, it is meanful to limit the distance of cellular user(use the same resource with D2D) to eNB.

As the frequency multiplex factor of LTE cell is nearly 1 ,the interference to cellular users at the edge of cell is serious, coupled with D2D communication, it become worse. So we take the method that the D2D users multiplex the resource which is used by the cellular users near the eNB, instead of any resource. It is priority radius to guarantee the outage probability of the cellular users. The interference in the whole system is analyzed as follows: 1 , the interference to cellular user is from D2D users, while the interference from cellular user which use the same resource in other cell is negligible;2、 the interference to $\mathrm{D} 2 \mathrm{D}$ receiver is from other $\mathrm{D} 2 \mathrm{D}$ transmitter and cellular user, both of them use the same resource. Most importantly, the priority radius should be set reasonable, the cellular user would 
be easy to outage if the radius is too large, similarly, it would be hardly to choose resource to multiplex if the radius is too small.

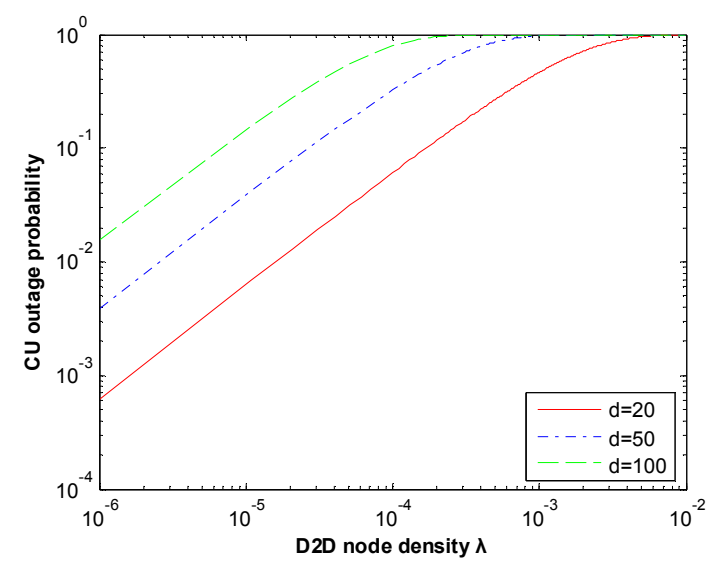

Fig.4. The CU outage probabitly in $d=20 、 50 、 100$

The outage probability and transmission capacity of D2D is analyzed. As the probability of Successful transmission is $\psi_{I_{D}}\left(V_{D} R_{D}^{\alpha}\right)=\prod_{j \in \phi} \psi_{I_{D j}}\left(V_{D} R_{D}^{\alpha}\right)$, there are two parts in $\phi$,one is from cellular system and the other is from other D2D transmitters. We denote the part from cellular system is follow, which is equal to formula(5):

$$
\begin{aligned}
\psi_{I D}\left(\mathrm{v}_{D} \mathrm{R}_{D}^{\alpha}\right) & =\exp \left\{-\lambda_{D} \int_{R^{2}} 1-E\left[e^{-V_{K} R_{D}^{\alpha} \delta|X|^{-\alpha}}\right] d x\right\} \\
& =\exp \left\{-\lambda_{D} \int_{R^{2}} \frac{V_{D} R_{D}^{\alpha} \delta|X|^{-\alpha}}{1+V_{D} R_{D}^{\alpha} \delta|X|^{-\alpha}} d x\right\} \\
& =\exp \left\{-C_{\alpha} R_{D}^{2} V_{D}^{2 / \alpha} \gamma_{D j} \lambda_{j}\right\}
\end{aligned}
$$

For the part from the cellular user, we proof as follow:

First, when the distance $\mathrm{D}_{\mathrm{CD}}$ between cellular user and D2D receiver is $l$ and that $l=\left(r^{2}+s^{2}-2 r d \cdot \cos \theta\right)^{1 / 2}$.the successful transmission probability of $\mathrm{D} 2 \mathrm{D}$ receiver is

$$
\begin{aligned}
& P\left(S I R_{D} \geq V_{D} \mid R_{D}=d_{D}, D_{C D}=l\right)=\operatorname{Pr} o\left(\frac{p_{D} \delta_{D D} d_{D}^{-\alpha}}{p_{C} \delta_{C D} l^{-\alpha}} \geq V_{D}\right) \\
= & \int_{0}^{\infty} P\left\{\delta_{D D} \geq \frac{I V_{D} d_{D}^{\alpha}}{p_{D}}\right\} f_{I}(I) d I \\
= & \int_{0}^{\infty} \frac{l^{\alpha}}{p_{C}} \exp \left(-\frac{l^{\alpha}}{p_{C}} I\right) \exp \left(-\frac{\theta d_{D}^{\alpha}}{p_{D}} I\right) d I \\
= & \frac{l^{\alpha}}{l^{\alpha}+\frac{p_{C} V_{D} d_{D}^{\alpha}}{p_{D}}}
\end{aligned}
$$

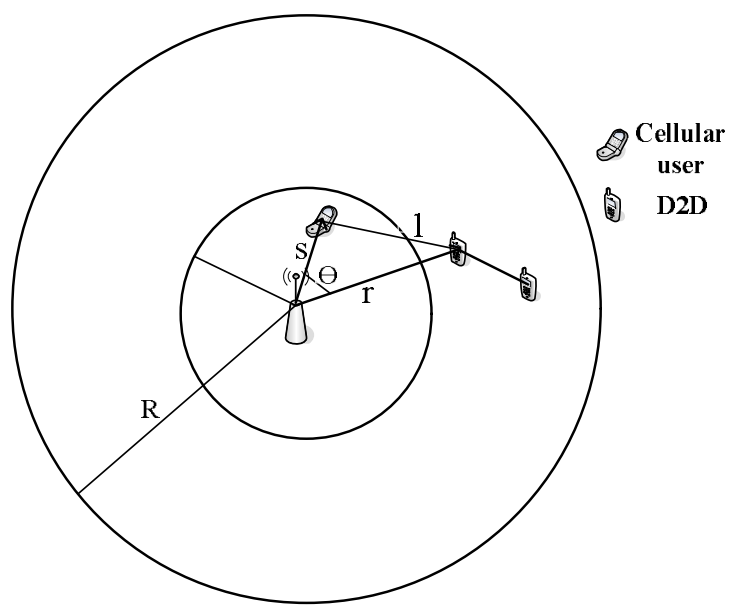

Fig.5. An example of priority radius, the D2D multiplex resources of cellular users which is inside radius D.

In the cellular networks, with different $l$ then the $\psi_{I C}\left(\mathrm{v}_{K} \mathrm{R}_{K}^{\alpha}\right)$ can be expressed as follow:

$$
\begin{aligned}
& \psi_{I C}\left(\mathrm{v}_{K} \mathrm{R}_{K}^{\alpha}\right)=P\left(S I R_{D} \geq v_{D}\right) \\
& =\int_{0}^{D} \int_{R^{2}} P\left(S I R_{D} \geq v_{D} \mid D_{C D}=l\right) \operatorname{Pr} o\left(D_{C D}=l\right) \operatorname{Pr} o\left(D_{C B}=s\right) d l d s \\
& =\int_{0}^{D} \int_{0}^{2 \pi} \int_{0}^{R} \frac{1}{2 \pi} \frac{l^{\alpha}}{l^{\alpha}+\frac{p_{C} \theta d_{D}^{\alpha}}{p_{D}}} \cdot \frac{2 r}{R^{2}} \cdot \frac{2 s}{D^{2}} d r d \theta d s
\end{aligned}
$$

Where $P_{C}$ and $P_{D}$ are power of cellular user and D2D transmitter, $R_{D}$ is the distance between $D 2 D$ pair, and $V_{D}$ is the target SIR.The priority radius is $\mathrm{D}, D_{C B}$ is the distance from cellular user to eNB, $\delta$ is the Rayleigh fading index. Then we can get the part of cellular, With the two part, the outage probability of D2D can be denoted for:

$$
\begin{aligned}
& P\left(\operatorname{SIR}_{D} \leq V_{D}\right) \\
= & 1-\exp \left\{-\frac{\pi^{2} d_{D}^{2} v_{D}^{2 / \alpha}}{2} \lambda_{D}\right\} \cdot \psi_{I C}\left(\mathrm{v}_{D} \mathrm{R}_{D}^{\alpha}\right)
\end{aligned}
$$

Similarly, the transmission capacity of the D2D can be denated by formula $(8)$. We set $R=500 \mathrm{D}=50 \mathrm{~V}_{\mathrm{D}}=10$, figure 6 is the theoretical and simulation outage probability of D2D when multiplex non-orthogonal resource, compared to Fig2 we can found that the outage probability is larger for the existence of cellular interference, while with the increasement of D2D density, the gap decreases. And figure7 is the theoretical and simulation transmission capacity of D2D.

\section{Summarize}

This paper studies D2D transmission capacity in cellular system, we calculate the outage probability and transmission capacity 
when D2D multiplex orthogonal and non-orthogonal resource respectively. Besides, we proposed a way to protect the successful communication of cellular user: the priority radius:D2D reuse resources of cellular users near the base station, which bring greater improvements in the outage probability of cellular users, and then calculated the outage probability and the transmission capacity of D2D

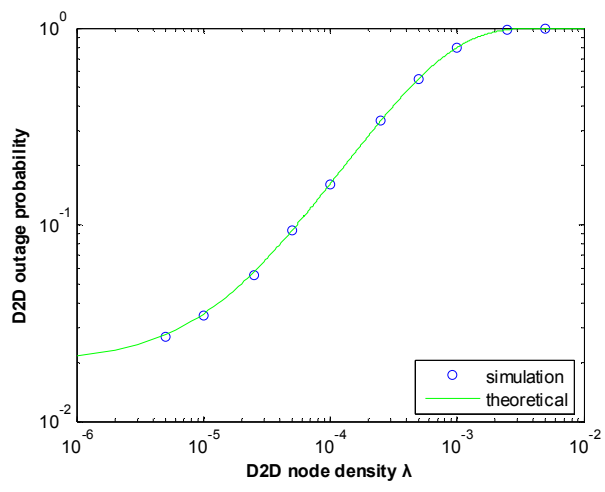

Fig.6. The D2D outage probability accroding to $\mathrm{D} 2 \mathrm{D}$ density, where $\alpha=4, \mathrm{R}=500, \mathrm{D}=50, \mathrm{~V}_{\mathrm{D}}=10$.

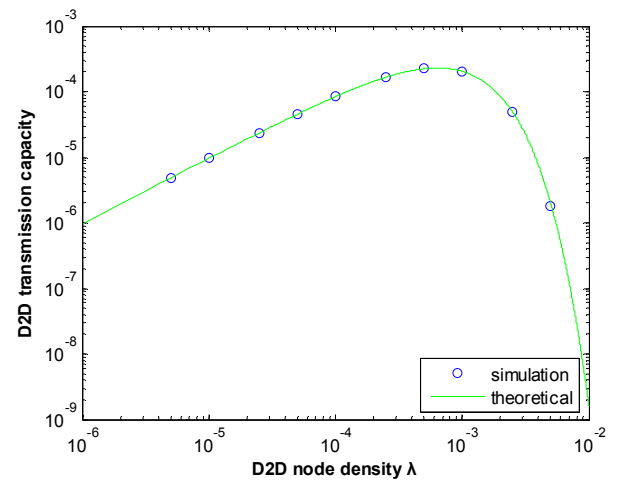

Fig.7. The D2D outage probability accroding to D2D density.

\section{References}

[1] 3GPP TR36.913v8.0.0.Requirements for further advancements for EUTRA.(2008)

[2] Chia-Hao Yu, Olav Tirkkonen, "Power optimization of device-to-device communication underlaying cellular communication", IEEE International Conference, pp.1-5, 2009.

[3] Klaus Doppler, Mika P. Rinne, "Device-to-Device communications; functional prospects for LTE-Advanced networks", IEEE International Conference, pp.1-6,2009

[4] A.Gjendemsjo,D.Gesbert,G.E.Oien, and S.G. Kiani. "Optimal power allocation and scheduling for two-cell capacity maximization".WiOpt, :1-6, 2006

[5] C.Yu,O.Tirkkonen,K.Doppler, and C.Ribeiro. "On the performance of Device-to-Device underlay communication with simple power control", IEEE VTC, Barcelona, Spain, pp.1-52009.
[6] Jänis P., Koivunen V., Ribeiro C., et.al, "Interference-aware resource allocation for device-to-device radio underlaying cellular networks", IEEE Vehicular Technology Conference (VTC),pp.1-5 2009

[7] Klaus Doppler, Chia-HaoYu, "Mode selection for Device-to-Device Communication underlaying an LTE-Advanced Network", IEEE Wireless Communications and Networking Conference(WCNC), pp.1-6, 2010.

[8] Kiran Vanganuru, Matthew Puzio, "Uplink System Capacity of a Cellular Network with Cooperative Mobile Relay", Wireless Telecommunications Symposium (WTS), pp. 1-7, 2011.

[9] S.Weber, X.Yang, J.Andrews, and G. deVeciana, "Transm Capacity of wireless adhoc networks with outage constraints," TIT,vol. 51,no.12, pp.4091-4102,2005.

[10] J.Lee,S.Lim,J.G.Andrews,D.Hong, “Achievable transmission capacity of secondary system in cognitive eradio networks," ICC,pp.1-5,2010.

[11] ZiyangLiu,TaoPeng,QianxiLu "Transmission capacity of D2D communication under heterogeneous networks with Dual Bands",PP.169174,CROWNCOM,2012.

[12] Jemin Lee; Andrews, J.G.; Daesik Hong "Spectrum-Sharing Transmission Capacity" ITOWC, VOL. 10, pp. 3053 - 3063,2011

[13] Kaibin Huang,Vincent K. N. Lau,and Yan Chen."Spectrum sharing between cellular and mobile ad hoc networks transmission capacity tradeoff'.IEEE Journal on Selected Areas in Communications. 27(7): 1256-1267.2009,

[14] Rahul Vaze."Transmission Capacity of Spectrum Sharing Ad Hoc Networks with Multiple Antennas". IEEE Transactions on Wireless Communications 10(7): 2334-2340 2011

[15] Rahul Vaze, Robert W. Heath Jr.:'Transmission Capacity of Ad-hoc Networks With Multiple Antennas Using Transmit Stream Adaptation and Interference Cancellation". IEEE Transactions on Information Theory 58 (2), pp: 780-792,2012

[16] Yuxin Chen, Jeffrey G. Andrews.” An upper bound on multi-hop transmission capacity with dynamic routing selection”. ISIT.pp. 17181722.2010 .

[17] F.Baccelli,B.Blaszczyszyn,andP.Muhlethaler,"An Aloha Protocol for Multihop Mobile Wireless Networks," IEEE Transactions on Information Theory, vol. 52, no.2,pp.421-436, 2006. 